\title{
Factors Affecting Hospital Reimbursements
}

\author{
Terry Jaqua ${ }^{1}$ and Ecler Jaqua ${ }^{2^{*}}$ \\ ${ }^{1}$ Trident University International, USA \\ ${ }^{2}$ Department of Family Medicine, Loma Linda University, USA
}

*Corresponding author: Ecler Jaqua, Department of Family Medicine, Loma Linda University, 1200 California Street, Suite 240, Redlands, CA 92374. Tel: 909-558-6688, E-mail: ejaqua@llu.edu

Citation: Terry Jaqua, Ecler Jaqua (2019) Factors Affecting Hospital Reimbursements. J Nurs Healthcare Manage 2: 103

Article history: Received: 19 January 2019, Accepted: 04 February 2019, Published: 06 February 2019

Hospital reimbursement is a critical issue in the healthcare sector. The performance of hospitals depends on the reimbursement programs, which are developed over time. There are many differences in the way patients are admitted and readmitted in the hospitals, and therefore, the reimbursements must be different. The length of treatment is another factor which affects the reimbursements in hospitals in such a way that the hospital which supports lengthy treatment of patients without referrals get more reimbursements compared to those which don't support lengthy procedures. The factors, which lead to lengthy treatments, include the need to guarantee full recovery for patients and the expertise of the health practitioners in a hospital.

Professional fees affect reimbursements by affecting the cost of medical services for patients. When physicians and other healthcare providers provide their services to patients, there are significant variations in the fees they charge for their services. These variations affect the revenues obtained by hospitals and therefore have an impact on hospital reimbursements. The facility fees also affect reimbursements by altering the cost of service delivery in hospitals. Facility fees deal with operational costs such as those involved in purchasing stationery and doing other practices [1]. In prospective payment systems, DRG weight is an essential consideration in calculating the extent of reimbursement, and it is the government, which comes up with such rates.

Documentation is vital in determining the levels of reimbursements in hospitals, and it considers the quality of the documents. Quality documentation means that more detailed strategies can be adopted to deal with information in the most economical way. Government regulations are essential in this process, and the health sector is required to comply with these regulations. The documentation should support care and ensure they are relatively accurate. The hospital personnel such as doctors must provide clear documentation, which shows their commitment to service provision in healthcare. The past medical history of patients is another factor to consider in guaranteeing reimbursement. When the medical history of a patient shows that the patient has been developing a specific condition for a long time, the rates of reimbursement for such a case are high.

From the cardiology clinic example, it is evident that some health conditions are considered for reimbursement more than others. There are severe ailments and health conditions in patients that should be managed promptly compare to others with requiring relatively less attention. Outpatient cases are not prioritized for reimbursements as compared to in-patient cases [2]. This is because; the costs related to inpatient cases are higher as compared with those of outpatients. The instances of insured patients are different from those who are not insured in calculating the levels of reimbursements. Most hospitals, which make more profits, serve commercially insured patients, and this means relatively higher payments. The types of insurance policies also matter in reimbursements and influence these rates.

In conclusion, reimbursements are essential in hospital settings, and they influence the levels of financial assistance to health institutions. The factors affecting payments include readmission, types of insurance policies held by patients, the medical conditions and past medical history of patients. The length of treatment is another factor that affects reimbursement, and it determines how the health facilities attend to patients and the expertise of physicians. Hospital-acquired conditions also affect reimbursement patterns and cause penalties to hospitals.

\section{References}

1. Murphy B (2016) Health Affairs: 5 factors that affect hospital profitability.

2. Deschenes S (2012) 5 market changes that will affect healthcare reimbursement. 